\title{
Diameter Dependence of Magnetic Properties of Co-based Metal Fibers ${ }^{1}$
}

\author{
Weiye Chen ${ }^{a}$, Guihong Geng ${ }^{a}$, Shuling Zhang ${ }^{b}$ (므 \\ ${ }^{a}$ School of Material Science and Engineering, North Nationalities University, Yinchuan, 750021, China \\ ${ }^{b}$ School of Mechanical \& Automotive Engineering, Qingdao University of Technology, Qingdao, 266520, China
}

Received: January 18, 2019; Revised: May 04, 2019; Accepted: May 19, 2019

\begin{abstract}
Naked $\mathrm{Co}_{68.25} \mathrm{Fe}_{4.5} \mathrm{Si}_{12.25} \mathrm{~B}_{15}$ metal fibers with diameter from $25 \mu \mathrm{m}$ to $100 \mu \mathrm{m}$ are produced by melt extraction method. Significant diameter dependence of magnetic properties is studied. Their microstructure and magnetic properties of hysteresis loops and giant magneto impedance (GMI) effect at frequencies from $0.1 \mathrm{MHz}$ to $13 \mathrm{MHz}$ are investigated. The results show that the coercivity increases with the diameter of fibers and the GMI effect is best in fiber with a diameter of $35 \mu \mathrm{m}$. It is found that the cooling rate of solidification decreases with the increase of fiber diameter. And fibers are amorphous with the diameter of $50 \mu \mathrm{m}$ and there are nanocrystallines in fibers with a diameter of $85 \mu \mathrm{m}$. The grain boundary blocks the magnetization process which makes lower circular permeability, larger coercivity and lower field sensitivity of GMI effect. The original microstructure of Co-based fibers decides their magnetic properties. Therefore, material design is important to improve the GMI effect in magnetic field sensor making.
\end{abstract}

Keywords: Metal fiber, GMI effect, hysteresis loops.

\section{Introduction}

Giant magneto-impedance (GMI) effect is a large and sensitive change in electrical impedance with the external dc magnetic field applied in a magnetic conductor ${ }^{1}$. It has attracted much attention from research community because of its potential applications in magnetic sensors and recording devices ${ }^{2}$. The GMI effect has found in amorphous ribbons, wires and films ${ }^{3,4}$. It is found that the Co-rich metallic fibers have good soft magnetic properties and excellent GMI effect ${ }^{5}$. The strong GMI effect of a piece of soft magnetic wire at a low dc magnetic field originates from the dependence of the circular magnetic permeability on the dc magnetic field and skin effect ${ }^{1}$. H. Chirac et al studied the impedance response of $\mathrm{Co}_{68.18} \mathrm{Fe}_{4.32} \mathrm{Si}_{12.5} \mathrm{~B}_{15}$ wires prepared by rotating water rapid quenching and found thinner wires had better GMI effect at low external field ${ }^{6}$. They also found the reduction of diameter by cold drawing produced a significant increase in GMI response ${ }^{6}$. H. Chirac et al also found the magnitude of the GMI effect was strongly dependent on the diameter of the metallic core and on the glass cover thickness through study on the GMI effect of glass-covered $\mathrm{Co}_{68.18} \mathrm{Fe}_{4.32} \mathrm{Si}_{12.5} \mathrm{~B}_{15}$ wires ${ }^{7}$. Naked wires prepared by melt extraction method are different from the hard drawing or glass-covered wires because there is no tensile stress induced by hard drawing process or the covered glass must be taken into account. The aim of our work is to study the magnetic properties of melt extraction $\mathrm{Co}_{68.25} \mathrm{Fe}_{4.5} \mathrm{Si}_{12.25} \mathrm{~B}_{15}$ amorphous wires with different diameters.

\section{Experimental}

$\mathrm{Co}_{68.25} \mathrm{Fe}_{4.5} \mathrm{Si}_{12.25} \mathrm{~B}_{15}$ amorphous wires were extracted directly from the melt alloy by a sharp rapidly rotating red copper wheel in an argon atmosphere ${ }^{8}$. The hysteresis loops of fiber with diameter of $45 \mu \mathrm{m}, 55 \mu \mathrm{m}, 60 \mu \mathrm{m}$ and $100 \mu \mathrm{m}$ were testes with induction method. The microstructure of fibers with $50 \mu \mathrm{m}$ and $80 \mu \mathrm{m}$ were analyzed by high resolution transmission electron microscopy, HRTEM. As-cast fibers of $25 \mu \mathrm{m}, 35 \mu \mathrm{m}, 44 \mu \mathrm{m}, 60 \mu \mathrm{m}$ in diameter and $20 \mathrm{~mm}$ in length were selected for impedance analysis with a distance between two voltages contacts of $15 \mathrm{~mm}$. Impedance $\mathrm{Z}$ was measured using Agient 4294A impedance analyzer at room temperature of about $25^{\circ} \mathrm{C}$. External dc axial magnetic field $H_{e x}$ was supplied by a pair of Helmholtz coils and the maximum field $H_{\text {max }}$ was 40 . The ac current amplitude was kept at $10 \mathrm{~mA}$ and the current frequency ranged from $0.1 \mathrm{MHz}$ to $15 \mathrm{MHz}$. The Helmholtz coils were kept perpendicular to the earth's magnetic field to avoid earth effect.

The impedance ratio can be expressed as:

$$
\Delta Z / Z(\%)=\left[Z\left(H_{e x}\right)-Z\left(H_{0}\right)\right] / Z\left(H_{0}\right) \times 100 \%
$$

Where $\mathrm{Z}\left(\mathrm{H}_{e x}\right)$ and $\mathrm{Z}\left(H_{0}\right)$ are impedance values at external magnetic field of $H_{e x}$ and zero.

\section{Results and Discussion}

The GMI amplitude as a function of the external magnetic field Hex is shown in Fig 1. The GMI response has different features with different diameters.

*e-mail: slzhang1229@163.com

${ }^{1}$ Key Scientific Research of North Nationalities University (Grant No. 2015KJ15) and National Natural Science Foundation of China (Grant No.51861031). 

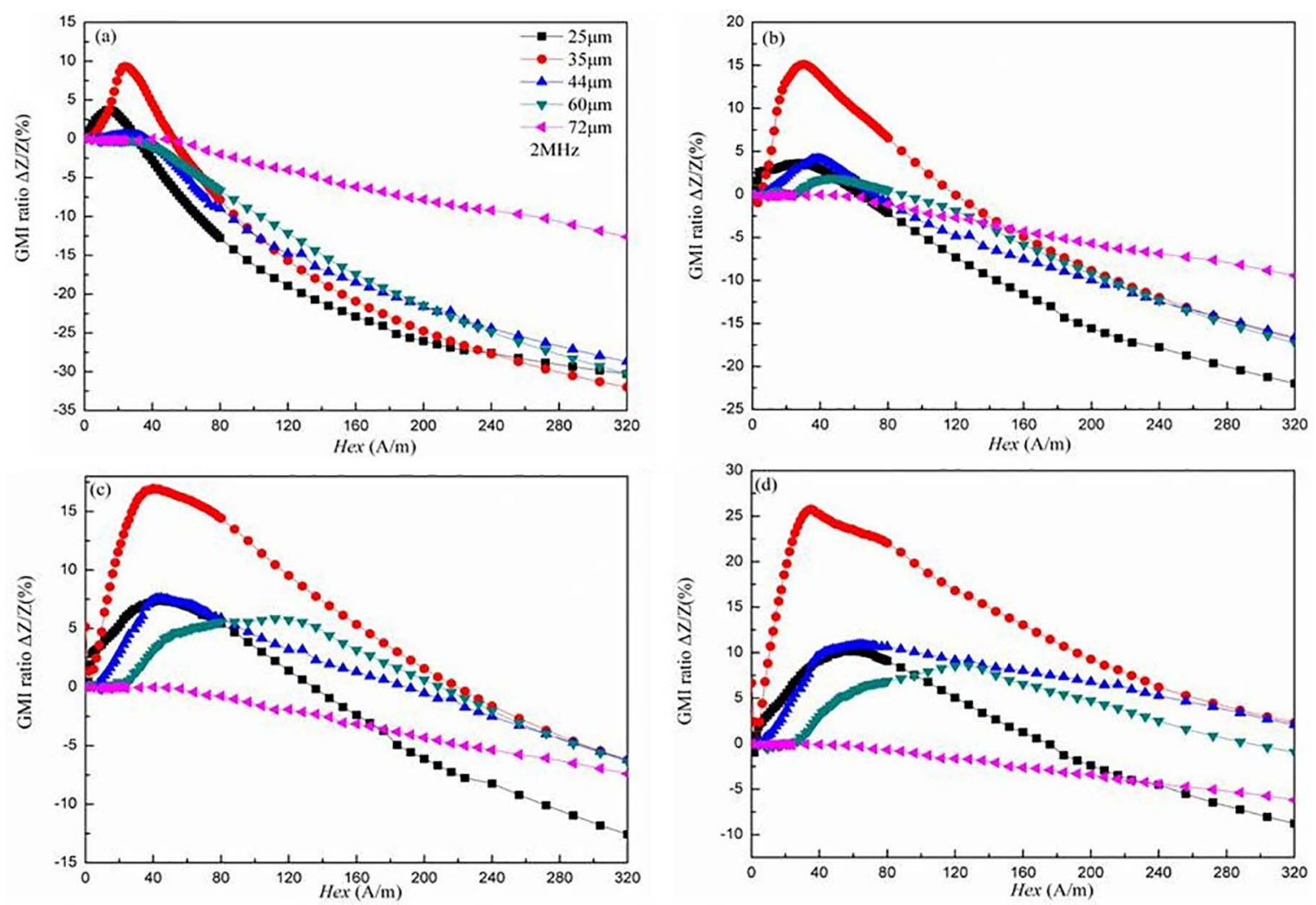

Figure 1. GMI profiles of $\mathrm{Co}_{68.25} \mathrm{Fe}_{4.5} \mathrm{Si}_{12.25} \mathrm{~B}_{15}$ metallic fibers with different diameters at different frequencies: (a) $2 \mathrm{MHz}$ (b) $5 \mathrm{MHz}$ (c) $10 \mathrm{MHz}(\mathrm{d}) 15 \mathrm{MHz}$.

GMI curves are all two-peak ones under different frequencies, fibers with diameter of $35 \mu \mathrm{m}$ has the strongest GMI effect, shown in Fig 2. The external field at peak impedance is named effective anisotropy field $H_{m}{ }^{9}$. The result shows that the filed $H_{m}$ increases gradually with the increase of diameter as shown in Fig 3. At $2 \mathrm{MHz}$, with the diameter increasing from $25 \mu \mathrm{m}$ to $60 \mu \mathrm{m}$, the corresponding $H_{m}$ increases from $14.4 \mathrm{~A} / \mathrm{m}$ to $27.2 \mathrm{~A} / \mathrm{m}$, and the $H_{m}$ increases from $27.2 \mathrm{~A} / \mathrm{m}$ to $46.4 \mathrm{~A} / \mathrm{m}$ at $10 \mathrm{MHz}$.

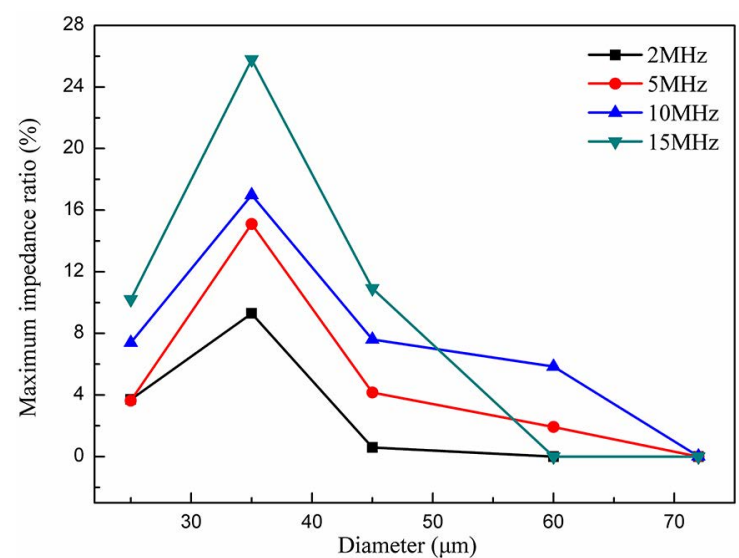

Figure 2. Diameter dependency of GMI effect of metallic fibers.

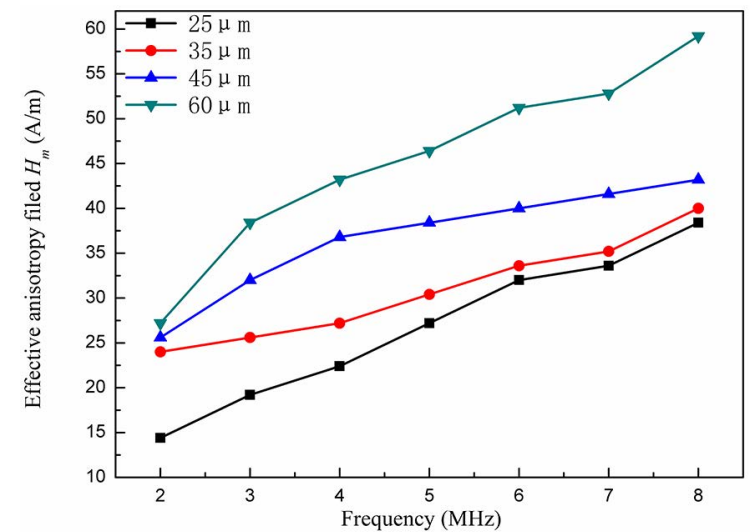

Figure 3. Frequency dependency on the effective anisotropy filed $H_{m}$ of fibers.

Fig. 4 presents the hysteresis loops of Co-based metal fibers with diameter of $45 \mu \mathrm{m}, 55 \mu \mathrm{m}, 60 \mu \mathrm{m}$ and $100 \mathrm{~m}$, respectively. With the increase of fiber diameter, the coercive field increases linearly. And the magnetization increases gradually which is mainly due to the increase of the quality and the greater the magnetic moment intensity.

Then the microstructure of fibers with diameter of $50 \mu \mathrm{m}$ and $85 \mu \mathrm{m}$ is investigated and shown in Fig 5. 


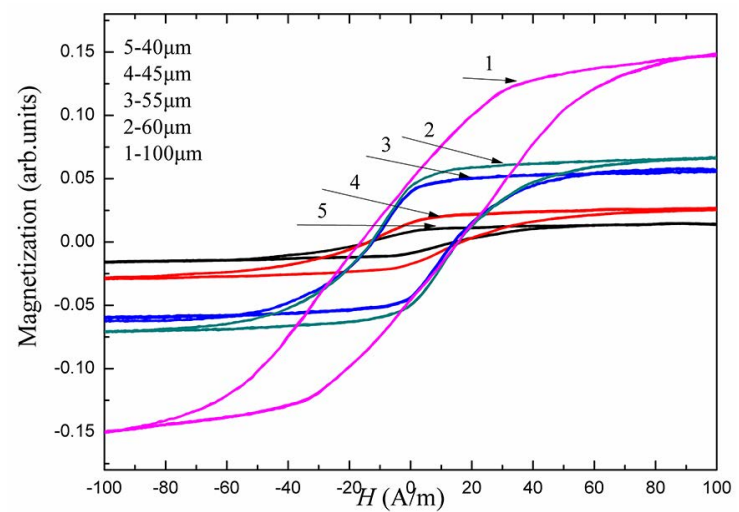

Figure 4. Hysteresis loops of $\mathrm{Co}_{6825} \mathrm{Fe}_{45} \mathrm{Si}_{1225} \mathrm{~B}_{15}$ metallic wires with different diameters.

There are short-range orders with a few of atoms and the microstructure is long range disorder, as shown in Fig 5(a). The short-range orders are formed by a central circular atomic structure or several close atoms packed into strips. The strip structure of single row atoms is irregular shape. After the fast Fourier transform, there is a diffraction ring or called a diffuse halo, no obvious crystal spots or nano ring appeared, which proves that the fiber is amorphous completely. That means during rapid solidification process, liquid atoms remain chaotic state and the fiber with a diameter of $50 \mu \mathrm{m}$ is amorphous one. Fig 5 (b) shows the microstructure of metal fibers of $85 \mu \mathrm{m}$ in diameter. After the fast Fourier transform, the diffraction ring is composed of several concentric circles, indicating the presence of nanophase in the amorphous matrix. There are long-range ordered regions, which are mainly circular and strip structures based on multi-layer long-range orders of atoms, as microcrystalline or nanocrystalline. The results show that the strip structure or circular structure is of $2 \mathrm{~nm} \times 4 \mathrm{~nm}$ in range with ruled atomic arrangement.
When the size of nanocrystalline is not large enough to block the magnetization process, the presence of small size nanocrystals is beneficial to the magnetization of fibers and to the sensitive GMI effect ${ }^{10,11}$.

It is proved that domains consist of an axial inner core domains and circular outer shell domains of this kind of Cobased fibers ${ }^{12,13}$. What's more, the thermal induced quenched-in stress in amorphous fibers is correlated with diameter ${ }^{7,14,15}$. The resulting domain configuration consists of a core axially magnetized and an outer shell which occupies an increase volume as diameter increases. Due to the increase of circular domains in thicker wires, the circular anisotropy field and permeability $\mu_{\Phi}$ increases and determines strong GMI effect. Two-peak GMI response appearance is correlated with the skin effect and magnetization process ${ }^{16,17}$. The magnetization driven by the ac current self-generated circular field decides circular permeability $\mu_{\Phi}$. In Co-based amorphous fibers the magnetization in the outer shell and the inner core contribute to the circular permeability ${ }^{18}$. As frequency increases, the skin effect gets strong and the domain wall displacement in the outer shell is damped by the eddy current. When the external field compensates for the anisotropy field, the circular permeability gets the largest values and the MI peak appears. It is investigated that the coercivity increases as diameter increase ${ }^{19}$ and the effective anisotropy field $H_{m}$ also increase as frequency ${ }^{20}$. For this reason, GMI effect will decreases when the diameter is over than $35 \mu \mathrm{m}$. Obviously, during rapid solidification process, a small number of atoms in the fibers of $85 \mu \mathrm{m}$ in diameter remain chaotic and disordered state as liquid atoms. Most atoms adjust their morphology to form regular long-range clusters and a certain amount of atoms even grow to be nanocrystals.

The difference in microstructure of the fibers with different diameters is related to the cooling rate of the fibers preparation.
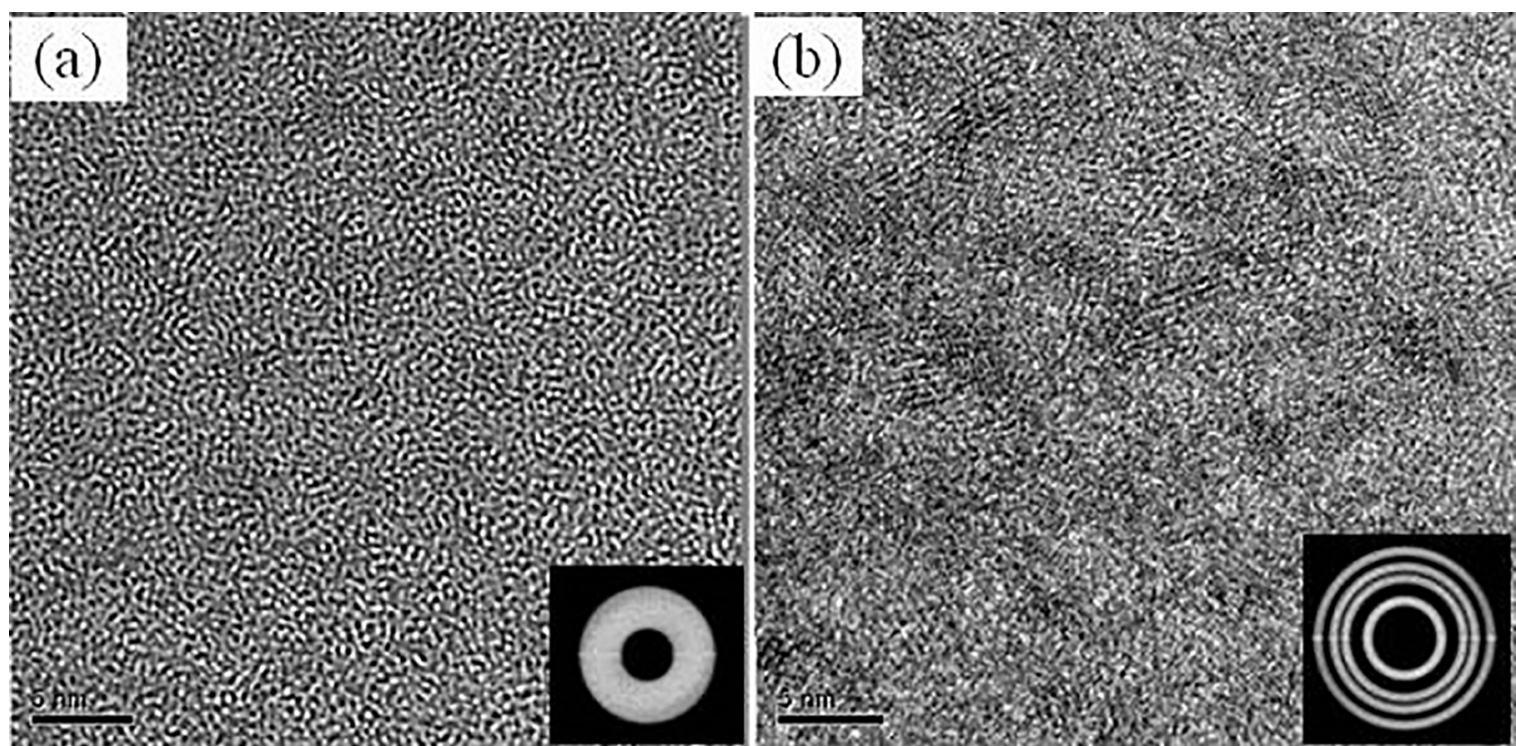

Figure 5. HRTEM maps of Microstructure and Fourier transform maps of metallic fibers in diameter: (a) $50 \mu \mathrm{m}$ (b) $85 \mu \mathrm{m}$. 
Under the same preparation conditions, the cooling rate of the alloy with the same component is determined only by the diameter of the fiber ${ }^{4,8,21}$. There is a reciprocal relationship between cooling rate and diameter. The increase of radius induces the decrease of cooling rate rapidly.

\section{Conclusions}

Through analysis the magnetic properties of naked $\mathrm{Co}_{68.25} \mathrm{Fe}_{4.5} \mathrm{Si}_{12.25} \mathrm{~B}_{15}$ melt extraction fibers of different diameters; it is found that their magnetic properties and GMI effect are significantly dependent on the diameter which decides the solidification cooling rate. And there are nanocrystals in the microstructures of fibers with a diameter of $85 \mu \mathrm{m}$ which induces larger coercivity. And the fibers of $50 \mu \mathrm{m}$ in diameter are completely amorphous with little coercivity. The increase of circular domains in thicker wires induces higher permeability $\mu_{\Phi}$ and better GMI effect. However, larger coercivity in thicker fibers also decreases the GMI effect. Consequently, GMI effect is best in fibers with a diameter of $35 \mu \mathrm{m}$.

\section{References}

1. Mohri K, Kohsawa T, Kawashima K, Yoshida H, Panina LV. Magneto-inductive effect (MI effect) in amorphous wires. IEEE Transactions on Magnetics. 1992;28(5):3150-3152.

2. Kamruzzaman M, Rahman IZ, Rahman MA. A review on magneto-impedance effect in amorphous magnetic materials. Journal of Materials Processing Technology. 2001;119(13):312-317.

3. Panina LV, Mohri K, Uchiyama T. Giant magneto-impedance (GMI) in amorphous wire, single layer film and sandwich film. Physica A: Statistical Mechanics and its Applications. 1997;241(1-2):429-438.

4. Phan MH, Peng HX. Giant magnetoimpedance materials: Fundamentals and applications. Progress in Materials Science. 2008;53(2):323-420.

5. Zhao Y, Hao H, Zhang Y. Preparation and giant magnetoimpedance behavior of Co-based amorphous wires. Intermetallics. 2013;42:62-67.

6. Chiriac H, Tibu M, Dobrea V. Magnetic properties of amorphous wires with different diameters. Journal of Magnetism and Magnetic Materials. 2005;290-291(Pt 2):1142-1145.

7. Chiriac H, Goian V, Corodeanu S. GMI Effect in Amorphous Glass Covered Microwires as a Function of the Internal Induced Stresses. IEEE Transactions on Magnetics. 2006;42(10):33593361.
8. Ström-Olsen J. Fine fibres by melt extraction. Materials Science and Engineering: A. 1994;178(1-2):239-243.

9. Vazquez M, Gomez-Polo C, Chen DX, Hernando A. Magnetic bistability of amorphous wires and sensor applications. IEEE Transactions on Magnetics. 1994;30(2):907-912.

10. Zhukov A, Ipatov M, Talaat A, Blanco JM, Churyukanova M, Granovsky A, et al. Engineering of Giant Magnetoimpedance Effect of Amorphous and Nanocrystalline Microwires. Journal of Superconductivity and Novel Magnetism. 2017;30(5):13591366.

11. Zhukova V, Ipatov M, Talaat A, Blanco JM, Churyukanova M, Zhukov A. Effect of stress annealing on magnetic properties and GMI effect of Co- and Fe-rich microwires. Journal of Alloys and Compounds. 2017;707:189-194.

12. Vazquez M, Chen DX. The magnetization reversal process in amorphous wires. IEEE Transactions on Magnetics. 1995;31(2):1229-1238.

13. Chen DX, Pascual L, Castano FJ, Vazquez M, Hernando A. Revised core-shell domain model for magnetostrictive amorphous wires. IEEE Transactions on Magnetics. 2001;37(2):994-1002.

14. Liu J, Malmhäll R, Arnberg L, Savage SJ. Theoretical analysis of residual stress effects on the magnetostrictive properties of amorphous wires. Journal of Applied Physics. 1990;67(9):42384240 .

15. Chiriac H, Óvári TA, Pop G. Internal stress distribution in glass-covered amorphous wires. Physical Review B: Condensed Matter. 1995;52:10104-10113

16. Xiao SQ, Liu YH, Yan SS, Dai YY, Zhang L, Mei LM. Giant magnetoimpedance and domain structure in $\mathrm{FeCuNbSiB}$ films and sandwiched films. Physical Review B: Condensed Matter. 2000;61(8):5734-5739.

17. Phan MH, Peng HX, Wisnom MR, Yu SC. Giant magnetoimpedance effect in ultrasoft FeAlSiBCuNb nanocomposites for sensor applications. Journal of Applied Physics. 2005;98(1):014316.

18. Mandal K, Sinha S, Kuma PA. Contributions to giant magnetoimpedance from different domian regions of $\mathrm{Co}_{68.15} \mathrm{Fe}_{4.35} \mathrm{Si}_{12.5} \mathrm{~B}_{15}$ amorphous wire. Journal of Applied Physics. 2006;99(3):033901.

19. Rudkowski P, Ström-Olsen JO. Frequency, magnetic field and size dependence of the magnetic properties of amorphous softmagnetic fibers. Journal of Magnetism and Magnetic Materials. 2002;249(1-2):85-88.

20. Phan MH, Peng HX, Yu SC, Vazquez M. Optimized giant magnetoimpedance effect in amorphous and nanocrystalline materials. Journal of Applied Physics. 2006;99(8):08C505.

21. Wang H, Xing D, Wang X, Sun J. Fabrication and Characterization of Melt-Extracted Co-Based Amorphous Wires. Metallurgical and Materials Transactions A. 2011;42(4):1103-1108. 